\title{
OS LIMITES DA MELHORIA CONTÍNUA: A ANÁLISE DO DESEMPENHO OPERACIONAL ANTES E APÓS TREINAMENTO
}

Fernanda de Sales Silva Rodrigues ' Isabel Cristina dos Santos ${ }^{2}$

Marilsa de Sá Rodrigues Tadeucci ${ }^{3}$

\section{Resumo}

Este artigo analisa os reflexos do treinamento operacional em ambiente de manufatura automatizado. A pesquisa tem caráter qualitativo, apoiada em análises documental e bibliográfica, e em entrevista semi-estruturada com 0 coordenador da operação. Observou-se o desempenho de um grupo de 35 operadores de máquinas de laminação a quente, a partir dos indicadores de produtividade, adotados pela empresa para todas as subsidiárias, em nível global. Os dados coletados referem-se ao primeiro semestre de 2005, ou seja, antes da aplicação do treinamento, e ao segundo semestre de 2005 , ou seja, após a aplicação do treinamento. O treinamento ocorreu em julho de 2005 , durante a parada prevista para manutenção das máquinas. Os dados operacionais obtidos são comparados aos indicadores de produtividade, definidos como padrão para a operação. Os resultados da pesquisa evidenciam que houve pouca influência do treinamento aplicado aos operadores sobre o desempenho dos principais indicadores, antes e após treinamento. Conclui-se que a homogeneidade do grupo, tempo e experiência na função são os principais responsáveis pelo alcance dos índices corporativos.

Palavras-chave: Treinamento; Desempenho; Manufatura.

\section{CONTINUOUS IMPROVEMENT BOUNDARIES: ANALYSIS OF OPERATIONAL PERFORMANCE BEFORE AND AFTER TRAINING ACTIONS}

\begin{abstract}
This paper aims to discuss the effectiveness of training initiatives in a high automatized manufacturing environment. The research procedures are based on documental analysis supported by a bibliographical research. It was performed a semi-structured interview with the operations coordinator in order to identify probable causes of variability in the manufacturing process It was identified a cluster of corporative productivity parameters which allowed to compare the results obtained in the first semester of 2005 to the results obtained in the second semester of the same year. Training actions were performed in July/2005. Real performance has been compared to the maximum limits, according to the corporative standards. Reductions causes are not seen as a consequence of the training applied but are most likely due to externalities conditions. Conclusions point out an existing operational improvement limit, which can be mostly defined by physical conditions than by human capabilities development.
\end{abstract}

Key words: Training; Performance; Manufacturing.

\section{INTRODUÇÃO}

A Gestão do Desempenho pautada em indicadores permite às organizações planejar e prover os recursos necessários para a melhoria contínua, o que, em tese, pode induzir o desempenho aos níveis superiores de alcance. Para tanto, segundo Takashina e Flores, ${ }^{(I)}$ é necessário estabelecer o conjunto de indicadores relevantes para o tipo de operação, de modo a validar o desempenho geral da organização. Por meio de indicadores, as organizações desdobram suas metas em vários níveis da estrutura organizacional, o que permite identificar suas necessidades de capacitação de mão-de-obra. Esse é o caso da empresa pesquisada.

\footnotetext{
'Mestre em Gestão e Desenvolvimento Regional da Universidade de Taubaté - UNITAU. Rua Expedicionário Ernesto Pereira, 225 - Portão 2 Centro - 12.020-330 - Taubaté - SP. fssrodrigues@yahoo.com.br

${ }^{2}$ Doutora em Engenharia pela EPUSP. Professora pesquisadora do Programa de Mestrado Profissional em Gestão e Desenvolvimento da UNITAU. Rua Expedicionário Ernesto Pereira, 225 - Portão 2 - Centro - 12.020-330 - Taubaté - SP. isa.santos.sjc@gmail.com

${ }^{3}$ Doutora em Administração pela Universidade Presbiteriana Mackenzie. Professora pesquisadora do Programa de Mestrado Profissional em Gestão e Desenvolvimento da UNITAU. Rua Expedicionário Ernesto Pereira, 225 - Portão 2 - Centro - 12.020-330 - Taubaté - SP. jmtadeucci@uol.com.br
} 
Vargas (apud MOURÃO)(2) conceitua treinamento e desenvolvimento como um processo único de "aquisição sistemática de conhecimentos capazes de provocar, a curto ou longo prazo, uma mudança na maneira de ser e de pensar do indivíduo".

Mourão ${ }^{(2)}$ complementa afirmando que estes conhecimentos devem ser capazes de provocar mudanças em um indivíduo, em suas funções de agir e pensar. Ou seja, não inclui na definição apenas a pura e simples aquisição do conhecimento, mas também a capacidade que este possui de provocar mudanças na vida de um indivíduo. A autora também apresenta a visão de Wexley que conceitua $\circ$ treinamento como uma ação organizacional planejada para facilitar a aprendizagem de comportamentos funcionais.

Para Milkovich e Boudreau, ${ }^{(3)}$ somente o treinamento é um processo sistemático de aquisição de conceitos, habilidades, regras e atitudes que busca $o$ alinhamento entre objetivos individuais do empregado e objetivos organizacionais. Milkovich e Boudreau ${ }^{(3)}$ caracterizam o desenvolvimento como um processo sistematizado, de longo prazo, com o objetivo de aperfeiçoar capacidades e motivações dos empregados para torná-los futuros membros valiosos da organização.

Considerando que o treinamento propicia o desenvolvimento, Donald Kirkpatrick, um dos mais referenciados autores em avaliação de treinamento (apud BOYLE e CROSBY) ${ }^{(4)}$ desenvolveu um sistema de avaliação de treinamento em quatro níveis de medição, a saber:

- Reação - reflete os sentimentos e opiniões dos treinandos sobre o treinamento. Essa avaliação é realizada logo após o treinamento e visa identificar as necessidades de melhoria dos materiais e métodos de instrução adotados;

- Aprendizado - este nível avalia a retenção de conhecimento transmitido no treinamento pelos treinandos. É normalmente realizada por meio de exames no meio e no final dos treinamentos. Segundo os autores, este nível é pouco utilizado nos processos de ensino realizados por organizações industriais;

- Aplicação ou Comportamento - avalia-se a transferência dos conhecimentos e das habilidades aprendidos no treinamento para o desempenho no trabalho; e

- Resultados - o nível que enfatiza a contribuição do treinamento para a realização da missão e objetivos organizacionais.

Dado haver grande permeabilidade entre fatores objetivos e subjetivos na análise do desempenho humano no trabalho, medir a influência do fator humano nos resultados da organização, especialmente em ambiente com alto nível de automação, é um desafio que tem se apresentado aos profissionais de Recursos Humanos, aos líderes de grupo e gestores.

Nas indústrias de capital aberto, subordinadas ao regime de governança corporativa, por exemplo, os investimentos de qualquer natureza estão sujeitos à aprovação colegiada, dos acionistas votantes, o que demanda a realização de um conjunto de análises e estudos de viabilidade que têm por objetivo avaliar a relação "custo - benefício" dos investimentos, e estimar o retorno financeiro ao longo do tempo. Desse modo, os investimentos tendem a ser definidos pelo benefício que trazem ao negócio e à continuidade da organização. Contudo, alguns desses benefícios esbarram nas fronteiras da capacidade de gerar grandes melhorias, ou saltos qualitativos. O presente artigo discute os limites da melhoria contínua.

\section{I.I Objetivos}

O objetivo geral deste trabalho é contribuir para a análise da efetividade das ações de treinamento em ambiente de manufatura.

Para atender ao objetivo geral, foram formulados os seguintes objetivos específicos: a) analisar os critérios e indicadores objetivos vinculados ao trabalho executado pelo grupo observado; e, b) avaliar a relação entre as ações de treinamento e a melhoria do desempenho, mediante a análise dos indicadores objetivos de produtividade.

\section{MATERIAL E MÉTODOS}

Com base nos objetivos propostos neste trabalho, esta pesquisa tem caráter descritivo, pois "se destina a relacionar características específicas de uma determinada população ou fenômeno, a partir das quais podem ser estabelecidas as relações entre as variáveis que compõem o objeto de estudo"(SANTOS). ${ }^{(5)}$ A abordagem em campo é qualitativa, pois, segundo Santos, ${ }^{(5)}$ visa a análise e compreensão dos efeitos psicossociais que um objeto de estudo tem sobre o seu ambiente e sua interação com a dimensão humana à medida que compara os elementos objetivos do desempenho aos elementos subjetivos da pesquisa de reação do grupo de empregados observados, também deste ambiente são os fatores que fundamentam o valor desta pesquisa.

Quanto aos procedimentos técnicos, a pesquisa teve caráter documental, apoiada em pesquisa bibliográfica, orientada para $\circ$ levantamento de conceitos e enunciados teóricos sobre indicadores de treinamento em ambiente de manufatura, na perspectiva de identificação dos indicadores objetivos do desempenho. Aos procedimentos adicionou-se, ainda, a observação sistemática, pela convivência cotidiana do pesquisador ao ambiente e grupo analisados.

A documentação indireta, utilizada no presente trabalho, baseada no levantamento de fontes de dados coletados por outras pessoas, elaborados ou não, é considerada por Marconi e Lakatos $^{(6)}$ como um dos mecanismos pelos quais se pode obter os dados relevantes para a pesquisa. No caso desta pesquisa, esta fase ocorreu a partir do levantamento e organização dos indicadores de desempenho e produtividade do grupo observado neste trabalho. 
Ainda, na fase de levantamento de dados, foi realizada entrevista semi-estruturada, com o coordenador do grupo observado que, além de coordenar a equipe, é especialista técnico da área. A entrevista e seu conteúdo foram autorizados pelo Comitê de Ética da Universidade de Taubaté, e tinham por objetivo obter esclarecimentos sobre as flutuações apontadas nos registros dos indicadores de desempenho do grupo, entre I de janeiro de 2005 e 3I de dezembro de 2005.

Foram formuladas dez questões gerais acerca da percepção e entendimento do entrevistado sobre relações entre o desempenho apontado e o treinamento aplicado ao grupo no período observado, e cinqüenta e oito questões específicas sobre os indicadores apontados no período observado.

Após a coleta dos dados, foram analisados os registros dos desempenhos do grupo observado com base em controles elaborados pela empresa em meio eletrônico e, em seguida, foi realizado o tratamento estatístico dos dados coletados a fim de se observar relações entre mudanças no desempenho evidentes nos registros $\mathrm{e}$ o treinamento aplicado. Os dados foram tabulados com a utilização do software MS-Excel e o tratamento estatístico com o software Minitab.

A amostra tomou um grupo de 35 operários do processo de Laminação a Quente, pertencente ao mesmo grupo de trabalho, em diferentes turnos de trabalho. Ainda, a amostra incluiu o coordenador deste grupo, em entrevista semi-estruturada.

\section{RESULTADOS}

Os indicadores de desempenho monitorados refletem os resultados do trabalho do grupo observado, cuja idade média dos indivíduos do grupo é de 36 anos, ou seja, oito pessoas com mais de 45 anos, dez pessoas com idade entre 35 e 44 anos, 15 pessoas com idade entre 25 e 34 anos e duas pessoas com 24 anos ou menos.

O tempo de casa médio é de 12 anos, sendo que mais de $50 \%$ do grupo é composto por pessoas com mais de dez anos de casa, o que evidencia o fato de não haver variações freqüentes no número de pessoas que compõem o grupo.

O tempo médio de exercício da função de operador é de seis anos, sendo o tempo maior de 18 anos e o menor de dois meses. A empresa, na figura do coordenador, considera que, para um operador estar apto a realizar as operações básicas dos equipamentos sob suas responsabilidades na área já mencionada, é necessário um período de experiência de, no mínimo, cinco anos.

O programa de treinamento considerado nesta pesquisa foi abrangente e abrigou em seu conteúdo programático as ocorrências mais freqüentemente observadas de desempenho abaixo do esperado e necessidades de aprimoramento e equiparação de métodos operacionais entre turmas. $O$ grupo observado atua no processo específico da área analisada e, para melhoria do desempenho, recebe o treinamento do processo observado, uma vez por ano, quando ocorre e em razão da parada anual para manutenção dos equipamentos envolvidos nas operações. Outros conteúdos de treinamento relacionados com o desempenho dos equipamentos ou atualização tecnológica são ministrados em módulo de educação à distância, que não é objeto da presente pesquisa.
Por determinação do coordenador deste grupo, durante a parada anual de manutenção dos equipamentos (período do ano no qual seqüencialmente os equipamentos da fábrica são desligados para manutenção), o grupo observado é reunido fora da fábrica para treinamento.

Em julho de 2005, o programa abordou as técnicas operacionais, e tinha como objetivo principal o nivelamento das práticas operacionais das diferentes turmas, em razão dos turnos de trabalho, e conseqüente melhoria e estabilidade no desempenho produtivo. O programa foi denominado de Princípios de Laminação a Quente e teve duração de dois dias e toda a grade curricular do treinamento foi ministrada por um especialista no processo de laminação.

$\mathrm{Na}$ Tabela I estão descritos os registros dos indicadores de desempenho do grupo observado, considerando o período I de janeiro de 2005 a 30 de junho de 2005.

Estão nesta tabela os registros dos indicadores de desempenho monitorados pelo grupo considerado nesta pesquisa, acompanhados de seu padrão (os limites máximos e mínimos) com exceção dos indicadores Horas Trabalhadas, Horas de Treinamento, Turn Over, Absenteísmo e Número de Acidentes, que não possuem índices padrões definidos.

$\mathrm{Na}$ Tabela 2 estão relacionados os mesmos indicadores, porém, registrados após a ação de treinamento operacional, entre I de julho de 2005 e 31 de dezembro de 2005.

Com o fito de encontrar diferenças nos indicadores de produtividade e desempenho do grupo observado após a ação de treinamento, realizou-se, com a utilização do software Minitab 14, o teste Two Sample T, também conhecido como teste de hipóteses. Neste teste, são comparadas as médias e desvios-padrão pré e pós-treinamento de cada indicador registrado nesta pesquisa, exceto para os indicadores Horas de Treinamento, Turnover, Absenteísmo e Número de Acidentes, considerando-se um índice de confiabilidade para o teste de $95 \%$.

A seguir, é apresentado o gráfico do único indicador monitorado que sofreu alterações estatisticamente significativas no período após a ação de treinamento. $O$ indicador refere-se ao consumo do óleo Tandemol, que é um insumo fundamental ao processo produtivo, cujo custo é o mais elevado em relação aos demais itens.

Na Figura I são apresentados o Individual Value e o Moving Range, sendo o primeiro a representação individual de cada resultado mensal do indicador e o segundo a representação do deslocamento ou variação do indicador pelo intervalo de confiança para a diferença entre as médias. 
Tabela I. Indicadores de desempenho observados entre janeiro e junho de 2005.

\begin{tabular}{|c|c|c|c|c|c|c|c|c|}
\hline & Acumulado & Ano & jan./05 & fev./05 & mar./05 & abr./05 & maio/05 & jun./05 \\
\hline \multirow[t]{2}{*}{ Índice rejeição } & Real & $1,13 \%$ & $0,62 \%$ & I,05\% & $1,27 \%$ & $2,21 \%$ & I,47\% & I,23\% \\
\hline & Lim. máximo & $1,00 \%$ & - & - & - & - & - & - \\
\hline \multirow[t]{2}{*}{ Índice reclassificação } & Real & $\mathrm{I}, 04 \%$ & $1,31 \%$ & $0,57 \%$ & $0,75 \%$ & $\mathrm{I}, 29 \%$ & $1,16 \%$ & $\mathrm{I}, 80 \%$ \\
\hline & Lim. máximo & $1,00 \%$ & - & - & - & - & - & - \\
\hline \multirow[t]{2}{*}{ Devolução interna } & Real & $0,58 \%$ & $0,24 \%$ & $0,23 \%$ & $0,58 \%$ & $0,59 \%$ & $0,35 \%$ & I,30\% \\
\hline & Lim. máximo & $0,50 \%$ & - & - & - & - & - & - \\
\hline \multirow[t]{2}{*}{ Consumo Tandemol* } & Real & 2,08 & 1,89 & 2,33 & 1,69 & 3,47 & 2,73 & 3,40 \\
\hline & Lim. máximo & 1,90 & - & - & - & - & - & - \\
\hline \multirow[t]{2}{*}{ Consumo Prosol* } & Real & 0,59 & 0,22 & 0,91 & 0,77 & 0,81 & 0,58 & 0,93 \\
\hline & Lim. máximo & 0,55 & - & - & - & - & - & - \\
\hline \multirow[t]{2}{*}{ Índice de utilização } & Real & 64,10 & 59,20 & 66,20 & 62,60 & 62,60 & 66,90 & 60,70 \\
\hline & Lim. mínimo & 68 & - & - & - & - & - & - \\
\hline Tempo de parada não & Real & 14,70 & 15 & 16,50 & 11,90 & 15,50 & 16,40 & 13,80 \\
\hline \multirow[t]{2}{*}{ previsto } & Lim. máximo & 14,30 & - & - & - & - & - & - \\
\hline & Real & - & 2,60 & 2,10 & 2,50 & 2,30 & 2,90 & 4,00 \\
\hline $\begin{array}{l}\text { Infrações operação } \\
\text { equipamentos móveis }\end{array}$ & Lim. máximo & $3,50 \%$ & - & - & - & - & - & - \\
\hline Horas trabalhadas & Real & 0 & 4791 & 4745 & 4374 & 4994 & 4676 & 4724 \\
\hline \multirow[t]{2}{*}{ Horas-extras } & Real & 0 & 711 & 407 & 529 & 980 & 795 & 571 \\
\hline & Lim. máximo & - & 479 & 475 & 437 & 499 & 468 & 472 \\
\hline Horas de treinamento & Real & - & 200 & 28 & 88 & 614 & 168 & 108 \\
\hline Rotatividade & Real & - & 0 & 0 & 0 & 0 & 0 & 0 \\
\hline Absenteísmo & Real & - & $\mathrm{I}, 58$ & 2,05 & 1,66 & 1,62 & 1,58 & 1,68 \\
\hline Número acidentes & Real & - & 0 & 0 & 0 & 0 & 0 & 0 \\
\hline
\end{tabular}

* óleo utilizado no equipamento de laminação a quente de chapas de alumínio

Tabela 2. Indicadores de desempenho observados entre julho e dezembro de 2005.

\begin{tabular}{|c|c|c|c|c|c|c|c|}
\hline & Acumulado & jul./05 & ago./05 & set./05 & out./05 & nov./05 & dez./05 \\
\hline \multirow[t]{2}{*}{ Índice rejeição } & Real & $1,06 \%$ & $1,00 \%$ & $0,83 \%$ & $0,72 \%$ & $0,67 \%$ & $1,47 \%$ \\
\hline & Lim. máximo & - & - & - & - & - & - \\
\hline \multirow[t]{2}{*}{ Índice reclassificação } & Real & $1,38 \%$ & $0,58 \%$ & $0,74 \%$ & $0,57 \%$ & $1,12 \%$ & $1,19 \%$ \\
\hline & Lim. máximo & - & - & - & - & - & - \\
\hline \multirow[t]{2}{*}{ Devolução interna } & Real & $0,42 \%$ & $0,74 \%$ & $0,80 \%$ & $0,88 \%$ & $0,40 \%$ & $0,46 \%$ \\
\hline & Lim. máximo & - & - & - & - & - & - \\
\hline \multirow[t]{2}{*}{ Consumo de Tandemol* } & Real & $\mathrm{I}, 74$ & $\mathrm{I}, 84$ & $\mathrm{I}, 54$ & $\mathrm{I}, 40$ & $\mathrm{I}, 72$ & $\mathrm{I}, 48$ \\
\hline & Lim. máximo & - & - & - & - & - & - \\
\hline \multirow[t]{2}{*}{ Consumo de Prosol* } & Real & 0,40 & 0,59 & $0,5 \mathrm{I}$ & 0,48 & 0,44 & 0,59 \\
\hline & Lim. máximo & - & - & - & - & - & - \\
\hline \multirow[t]{2}{*}{ Índice de utilização } & Real & 58,70 & 68 & 68,70 & 61,80 & 67,70 & 66,80 \\
\hline & Lim. mínimo & - & - & - & - & - & - \\
\hline Tempo de Parada Não & Real & 15,30 & 13,90 & 15,50 & 12,30 & 13 & 16,90 \\
\hline Previsto & Lim. máximo & - & - & - & - & - & - \\
\hline Infrações na operação & Real & 2,70 & 3,00 & 3,20 & 2,40 & $\mathrm{I}, 40$ & 1,60 \\
\hline equipamentos móveis & Lim. máximo & - & - & - & - & - & - \\
\hline Horas trabalhadas & Real & 4817 & 4835 & 4763 & 4540 & 4812 & 4419 \\
\hline \multirow[t]{2}{*}{ Horas-extras } & Real & 677 & 443 & 225 & 414 & 672 & 260 \\
\hline & Lim. máximo & 482 & 483 & 476 & 454 & 481 & 442 \\
\hline Horas de treinamento & Real & 228 & 316 & 225 & 216 & 232 & 167 \\
\hline Turn over & Real & 0 & 0 & 0 & 0 & 0 & 0 \\
\hline Absenteísmo & Real & $\mathrm{I}, 84$ & 1,58 & I,7I & $|, 5|$ & 1,94 & 2,07 \\
\hline Número acidentes & Real & 0 & 0 & 1 & 0 & 0 & 0 \\
\hline
\end{tabular}

* óleo utilizado no equipamento de laminação a quente de chapas de alumínio

No teste deste indicador comprovou-se variação estatisticamente válida para melhor no período após a ação de treinamento. $A$ média dos resultados antes da ação de treinamento era de 2,585 e no período após a ação foi de $I, 620$. O cálculo do $P$-value para este indicador teve como resultado 0,028 , portanto menor que 0,05 , o que indica que os valores mensais deste indicador, no período após a ação de treinamento, ficaram fora dos limites de não-significância da curva de distribuição normal.

\section{DISCUSSÃO}

Observou-se que, em consonância aos conceitos apresentados, as ações de treinamento e desenvolvimento do grupo observado tiveram como objetivo específico o aperfeiçoamento dos resultados da operação de laminação a quente. 
Análise: Consumo Tandemol
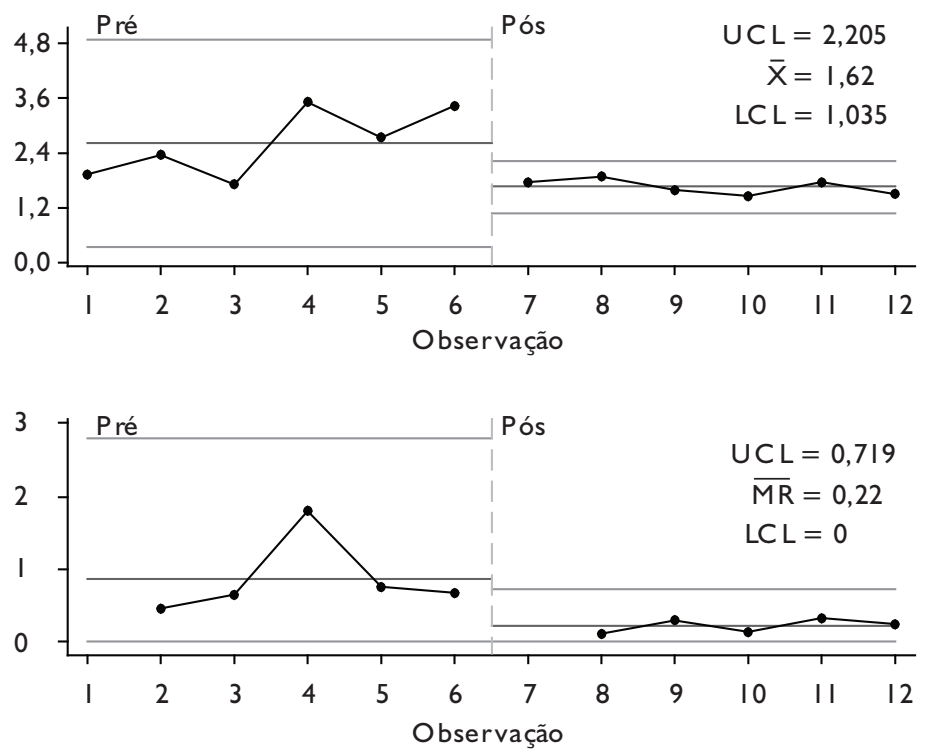

Figura I. Consumo de Tandemol.

Contudo, sendo essa uma operação tradicional, estabilizada pela aplicação de padrões de desempenho e do uso de tecnologia de ponta, não surpreende o fato da baixa variação nos índices analisados, na condição antes e após treinamento.

Limites estreitos definem o espaço de intervenção dos indivíduos, o que relativiza o postulado da melhoria contínua em processos com semelhante nível de automação, fundamentados quase que exclusivamente no comportamento e nas escolhas dos indivíduos com relação à aplicação do conhecimento.

A maturidade do grupo em relação à operação observada sugere a experimentação cotidiana de eventos operacionais intervenientes no resultado geral da operação. Desse modo, o treinamento aplicado também pode ser visto como uma oportunidade de compartilhamento das lições aprendidas com a prática e, com isso, eliminar a repetição de ocorrências indesejáveis no processo produtivo, aprimorando os métodos de trabalho entre operadores.
Os resultados obtidos na pesquisa mostram-se, portanto, coerentes com o conceito apresentado por Milkovich e Boudreau, ${ }^{(3)}$ que consideram o treinamento um processo sistemático de aquisição de conceitos, habilidades, regras e atitudes, que busca $o$ alinhamento entre objetivos do empregado e objetivos organizacionais.

Observando-se o modelo proposto por Kirkpatrick (apud Boyle e Crosby) ${ }^{(4)}$ para avaliação de programas de treinamento, seu quarto nível descreve a relação do método de medição e monitoramento do desempenho utilizado pela empresa observada nesta pesquisa e seu quarto nível de avaliação - o de resultados.

\section{CONCLUSÃO}

O controle e a medição dos resultados de treinamento não devem ser avaliados exclusivamente pelos indicadores que os originaram, uma vez que variáveis aleatórias podem afetar o resultado dos indicadores após o treinamento. Na pesquisa não foi possível isolar os impactos diretos do treinamento no desempenho do grupo em qualquer de seus indicadores. A empresa não identificou, até $o$ momento final da pesquisa, quais seriam as variáveis subjetivas ou ambientais que afetam cada um dos indicadores de desempenho. Um exemplo é o índice de Consumo de Tandemol, o único a sofrer alterações significativas após o treinamento. A melhoria neste indicador, mesmo mantidas as condições primárias de produção, pode ter sido influenciada por outras variáveis, como temperatura média do ambiente no período, número menor de contaminações do insumo, entre outras.

No caso específico deste trabalho, a ação de treinamento objetivava melhorar o desempenho do grupo nos processos monitorados por indicadores corporativos. Porém, não é possível afirmar que as alterações estatisticamente significativas nos indicadores tenham ocorrido exclusivamente em função do treinamento.

\section{REFERÊNCIAS}

I TAKASHINA, N.T.; FLORES, M.C.X. Indicadores da qualidade e do desempenho. Rio de Janeiro: Qualitymark, 1996.

2 MOURÃO, L. Avaliação de programas públicos de treinamento: um estudo sobre o impacto no trabalho e na geração de empregos. 2004. 210 f. Tese (Doutorado em Psicologia) - Instituto de Psicologia da Universidade Nacional de Brasília, 2004.

3 MILKOVICH, G.T.; BOUDREAU, J.W. Administração de recursos humanos. São Paulo: Atlas, 2000.

4 BOYLE, M.A.; CROSBY, R. Academic program evaluation. Journal of Industrial Teacher Education, 1997. Disponível em: <http://scholar.lib.vt.edu/ejournals/JITE/v34n3/Atlssue.html/Kirkpatrick>. Acesso em I3 fev 2007.

5 SANTOS, I.C. Um modelo estruturado de gestão do conhecimento em indústrias de base tecnológica. 2004. 185 f. Tese (Doutorado em Engenharia) - Escola Politécnica da Universidade de São Paulo, 2004.

6 MARCONI, M.A.; LAKATOS, E.M. Metodologia do trabalho científico. 6. ed. São Paulo: Atlas, 200 I.

Recebido em: 9/06/08

Aceito em: 6/10/08

Proveniente de: CONGRESSO ANUAL DA ABM, 62., 2007, Vitória, ES. São Paulo: ABM, 2007. 